\title{
Association of cytokine gene polymorphisms with osteoarthritis susceptibility
}

\author{
OTILIA CONSTANTINA ROGOVEANU ${ }^{1 *}$, DANIELA CALINA $^{2 *}$, MIHAI GABRIEL CUCU $^{3}$, FLORIN BURADA $^{3}$, \\ ANCA OANA DOCEA ${ }^{4}$, SIMONA SOSOI $^{3}$, EMILIAN STEFAN $^{5}$, MIHAI IOANA $^{3}$ and EMILIA BURADA ${ }^{3,6}$ \\ ${ }^{1}$ Department of Physical Medicine and Rehabilitation; ${ }^{2}$ Department of Clinical Pharmacy; \\ ${ }^{3}$ Human Genomics Laboratory; ${ }^{4}$ Department of Toxicology, University of Medicine and Pharmacy of Craiova, \\ 200349 Craiova; ${ }^{5}$ Clinic of Orthopaedics and Traumatology, Clinical Hospital CF2, 011464 Bucharest; \\ ${ }^{6}$ Department of Physiology, University of Medicine and Pharmacy of Craiova, 200349 Craiova, Romania
}

Received December 18, 2017; Accepted June 20, 2018

DOI: $10.3892 /$ etm.2018.6477

\begin{abstract}
Osteoarthritis (OA) is a multifactorial disease characterized by low-grade inflammatory processes that are mediated initially by the cells and factors of the innate immune system. In addition to their key role in inflammation, cytokines contribute to the pathogenesis of OA through angiogenesis and chemotaxis. The purpose of the present case-control study was to investigate a possible association of four cytokine single nucleotide polymorphisms (SNPs), IL-4R -3223C >T (rs2057768), IL-8 -251T>A (rs4073), IL-10 $-1082 \mathrm{~A}>\mathrm{G}(\mathrm{rs} 1800896)$ and TNF -A-308G $>$ A (rs1800629) with OA susceptibility. Genomic DNA was isolated from blood samples collected from 305 Romanian subjects (90 patients with OA and 215 controls) and the genotyping of the SNPs was performed by TaqMan allelic discrimination polymerase chain reaction using predesigned assays. Our data indicated a significant association for IL-4R rs2057768 C>T SNP. The subjects that carried the $\mathrm{CT}$ genotype were at a higher risk for OA (OR 2.03, 95\% CI: 1.21-3.42, P=0.007) compared with those that had the $\mathrm{CC}$ genotype. Furthermore, the carriers of the $\mathrm{T}$ allele were at a 1.9 fold higher risk for OA (OR 1.92; $95 \% \mathrm{CI}, 1.17-3.17$; $\mathrm{P}=0.009$ ) in a dominant model. The association remained significant only for knee OA in the subgroups analysis. No correlations were observed between the other
\end{abstract}

Correspondence to: Dr Florin Burada, Human Genomics Laboratory, University of Medicine and Pharmacy of Craiova, 2 Petru Rares, 200349 Craiova, Romania

E-mail: buradaflorin@gmail.com

Dr Anca Oana Docea, Department of Toxicology, University of Medicine and Pharmacy of Craiova, 2 Petru Rares, 200349 Craiova, Romania

E-mail: ancadocea@gmail.com

*Contributed equally

Key words: cytokine, genotype, inflammation, osteoarthritis, single nucleotide polymorphism remaining SNPs and OA. The results suggested that the IL-4R rs2057768 SNP could contribute to OA susceptibility in the Romanian population, providing novel evidence for the involvement of IL-4/IL-4R pair in the pathogenesis of OA.

\section{Introduction}

Osteoarthritis (OA) is a chronic disease characterized by progressive degradation and loss of articularcartilage. Although the disease present in various joints, the weight-bearing joints such as knees and hips are commonly affected. Furthermore, alterations in other joint tissues such as ligaments, synovium and subchondral bone are usually noted in OA $(1,2)$. Generally, the symptoms develop gradually over time and the loss of cartilage can lead to decreased joint space, stiffness, pain with increasing loss of function and disability, and finally to the need for surgical joint replacement (3). Nowadays, OA is not only viewed as a degenerative disease of cartilage and different risk factors were associated with OA, including genetic predisposition, sex, obesity, age, diet, occupation, metabolic syndromes, injury and mechanical stress $(1,4,5)$. Accumulating evidence indicates that inflammation has a critical role in OA pathogenesis (6), and recent findings show that the development of OA is in notably driven by low-grade inflammatory processes and mediated mainly by the innate immune system (7). The inflammatory response was identified as the key component, which promoted synovitis as well as progression of cartilage and bone destruction in OA via the secretion of chemokines, cytokines and other molecules, which can be detected in the synovial fluids (8-10). In addition to their evident role in inflammation, cytokines can contribute to the pathogenesis of OA through angiogenesis and chemotaxis $(11,12)$. OA can take years and even decades to develop and radiography is routinely used to help in the diagnosis of OA. However, symptoms often occur before the onset of any radiographic abnormality and further work should be focused on the identification of new biological markers as early indicators of OA risk.

Based on this rational, we have genotyped four single nucleotide polymorphisms (SNPs) located in the promoter region of the following cytokine genes: IL-4R $-3223 \mathrm{C}>\mathrm{T}$ 
(rs2057768), IL-8 -251T>A (rs4073), IL-10 -1082A > G (rs1800896) and TNF-A -308G>A (rs1800629). This study was performed in a Romanian population (Eastern European population) in order to establish whether these cytokine gene SNPs are associated with OA susceptibility in this region.

\section{Materials and methods}

Subjects. In the current hospital-based case-control study we included 305 Romanian subjects (90 patients diagnosed with $\mathrm{OA}$ and 215 controls). All OA cases were diagnosed based on clinical and radiographic signs, arthroscopic or MRI findings with Kellgren-Lawrence grade 2 or more. The patients with history of trauma or skeletal defects were excluded. Matched controls of the same ethnic and geographical origins were recruited among unrelated volunteers. The controls were admitted in the Clinical Hospital CF2 from Bucharest and in the Emergency Clinical County Hospital of Craiova, Romania. The subjects with a positive history of tumor, autoimmune, other inflammatory or chronic infectious symptoms were excluded. Written informed consent from all the participants was obtained and the study was approved by the Ethics Committee of the University of Medicine and Pharmacy of Craiova (Craiova, Romania).

SNPs genotyping. Total genomic DNA was isolated from the peripheral blood leukocytes using the Wizard Genomic DNA Purification kit (Promega Corporation, Madison, WI, USA) following the manufacturer's protocol. DNA concentration and purity were determined with UV spectrophotometry. The identification of SNPs was performed by TaqMan allelic discrimination real-time PCR. Validated TaqMan SNP genotyping assays were obtained from Applied Biosystems (Foster City, CA, USA): IL-4R -3223C >T (rs2057768, assay C_2769607_10); IL-8 -251T>A (rs4073, assay C_11748116_10); IL-10 -1082A $>$ G (rs1800896, assay C_1747360_10) and TNF-A -308G $>$ A (rs1800629, assay C_7514879_10). The procedures for the PCR reactions and quality control samples have been described previously (13).

Statistical analysis. The deviation from Hardy-Weinberg equilibrium was tested among controls by $\chi^{2}$ test. The demographic data between groups were compared using the $\chi^{2}$ test for sex and the Student's t-test for age. Using unconditional logistic regression analysis under codominant and dominant models, we have calculated the differences in genotype distributions and minor allele frequencies among OA patients and controls based on Odds ratios (OR) and 95\% confidence intervals (CIs). The reference group included the subjects that were homozygous for the most common allele. A stratified analysis by joint location was further performed aside from the overall association analysis. SPSS software package (v.17.0; SPSS Inc., Chicago, IL, USA) was used for data analysis and a P-value of less than 0.05 indicated a statistically significant difference.

\section{Results}

Subjects characteristics. A total of 90 OA patients (63 women and 27 men, mean age 64.12 years, with a body mass index $\leq 27$ ) and 215 healthy controls (150 women and 65 men, mean age
Table I. Baseline characteristics of the patients and controls.

\begin{tabular}{lccc}
\hline Characteristic & $\begin{array}{c}\text { Osteoarthritis } \\
\text { cases }(\mathrm{n}=90)\end{array}$ & $\begin{array}{c}\text { Control } \\
(\mathrm{n}=215)\end{array}$ & P-value \\
\hline Male/female & $63 / 27$ & $150 / 65$ & 0.97 \\
Age (years) & 64.12 & 62.69 & 0.26 \\
Location & & & \\
Knee & 54 & - & - \\
Hip & 36 & - & - \\
\hline
\end{tabular}

62.69 years) were included in the present study. In 54 cases the location was within the knee and in 36 cases within the hip joint. The age and sex distributions of the groups were comparable (Table I).

Cytokine SNPs and risk of overall OA. For each polymorphism, the genotype distributions were consistent with those predicted by the Hardy-Weinberg equilibrium. A significant association was observed for IL-4R $-3223 \mathrm{C}>\mathrm{T}$ (rs2057768) SNP. The subjects carrying $\mathrm{CT}$ genotype were at a higher risk for OA (OR 2.03; 95\% CI: 1.21-3.42; P=0.007) compared with the more frequently encountered $\mathrm{CC}$ genotype. Furthermore, the carriers of $\mathrm{T}$ allele were at a 1.9 fold elevated risk for $\mathrm{OA}$ in a dominant model (OR 1.92; 95\% CI, 1.17-3.17; $\mathrm{P}=0.009$ ). The $\mathrm{T}$ allele was significantly more frequent in patients with $\mathrm{OA}$ when the allele frequencies were assessed (OR 1.51; 95\% CI, 1.03-2.21; $\mathrm{P}=0.035$ ).

No correlation was noted between OA cases and controls with regard to IL-8 -251AA, IL-10 -1082GG and TNF-A $-308 \mathrm{AG}$ genotypes and overall OA risk (Table II). In addition, the carriers of IL-10 -1082 G, IL-8 -251A and TNF-A -308A allele were not associated with an increased risk of OA in a dominant model.

Risk of knee and hip OA by genotype. In a stratified analysis, the only association between $\mathrm{OA}$ and cytokine polymorphisms was found for IL-4R $-3223 \mathrm{C}>\mathrm{T}$ polymorphism and was restricted to the knee OA cases. The T carriers exhibited an increased risk (OR 2.31; 95\% CI, 1.26-4.26) (Table III; Fig. 1). No significant differences were observed between knee or hip $\mathrm{OA}$ and controls in the subgroups analysis for the remaining SNPs (Table III; Fig. 2).

\section{Discussion}

In the present observational study, we assessed whether the four promoter SNPs located in the cytokine genes influence the risk of $\mathrm{OA}$ in the Romanian population. The polymorphisms were selected based on multiple previous reports that have demonstrated quantitative differences in the transcription and/or expression, and their involvement in other multifactorial diseases, where the inflammatory process plays an important role in pathogenesis.

From the tested SNPs, the only association was detected for IL-4R rs2057768 C>T SNP. This gene encodes the alpha chain of the interleukin- 4 receptor that can bind the anti-inflammatory cytokine IL-4. Previous research investigated the association 
Table II. Genotype frequencies for cytokine polymorphisms in cases and controls and their association with risk of osteoarthritis.

\begin{tabular}{|c|c|c|c|c|}
\hline Polymorphism & Osteoarthritis (n=90) (\%) & Control $(n=215)(\%)$ & OR $(95 \% \mathrm{CI})$ & P-value \\
\hline \multicolumn{5}{|l|}{ IL-4R $-3223 C>T$} \\
\hline $\mathrm{CC}$ & $39(43.33)$ & $128(59.54)$ & Reference & - \\
\hline $\mathrm{CT}$ & $44(48.89)$ & $71(33.02)$ & $2.03(1.21-3.42)$ & 0.007 \\
\hline TT & $7(7.78)$ & $16(7.44)$ & $1.44(0.55-3.74)$ & 0.457 \\
\hline $\mathrm{T}$ carriers & $51(56.67)$ & $87(40.46)$ & $1.92(1.17-3.17)$ & 0.009 \\
\hline \multicolumn{5}{|l|}{ IL-8 -251T>A } \\
\hline $\mathrm{TT}$ & $22(24.44)$ & $74(34.42)$ & Reference & - \\
\hline TA & $51(56.67)$ & $103(47.91)$ & $1.67(0.93-2.98)$ & 0.084 \\
\hline AA & $17(18.89)$ & $38(17.67)$ & $1.51(0.72-3.17)$ & 0.280 \\
\hline A carriers & $68(75.56)$ & $141(65.58)$ & $1.62(0.93-2.83)$ & 0.097 \\
\hline \multicolumn{5}{|c|}{ IL-10 -1082 A>G } \\
\hline AA & $33(36.67)$ & $81(37.67)$ & Reference & - \\
\hline $\mathrm{AG}$ & $43(47.78)$ & $103(47.91)$ & $1.02(0.60-1.76)$ & 0.929 \\
\hline GG & $14(15.56)$ & $31(14.42)$ & $1.11(0.52-2.35)$ & 0.787 \\
\hline G carriers & $57(63.33)$ & $134(62.33)$ & $1.04(0.63-1.74)$ & 0.868 \\
\hline \multicolumn{5}{|c|}{ TNF-A -308 G>A } \\
\hline GG & $73(81.11)$ & $173(80.47)$ & Reference & - \\
\hline GA & $17(18.89)$ & $40(18.60)$ & $1.07(0.54-1.89)$ & 0.982 \\
\hline AA & $0(0.00)$ & $2(0.93)$ & - & - \\
\hline A carriers & $17(20.14)$ & $42(19.53)$ & $0.96(0.51-1.79)$ & 0.896 \\
\hline
\end{tabular}

OR, odds ratio; CI, confidence interval; TNF, tumor necrosis factor; IL, interleukin.

Table III. Comparative analysis between genotype frequencies and the risk of osteoarthritis in the joint location subgroups.

\begin{tabular}{|c|c|c|c|c|c|c|}
\hline Polymorphism & Knee, $\mathrm{n}=54(\%)$ & OR $(95 \% \mathrm{CI})$ & P-value & Hip, n=36 (\%) & OR $(95 \%$ CI $)$ & P-value \\
\hline \multicolumn{7}{|c|}{ IL-4R -3223C>T } \\
\hline $\mathrm{CC}$ & $21(38.89)$ & Reference & & $18(50.00)$ & Reference & \\
\hline $\mathrm{CT}$ & $31(57.41)$ & $2.66(1.42-4.97)$ & 0.002 & $13(36.11)$ & $1.30(0.60-2.81)$ & 0.501 \\
\hline TT & $2(3.70)$ & $0.76(0.16-3.56)$ & 0.728 & $5(13.89)$ & $2.22(0.73-6.80)$ & 0.153 \\
\hline $\mathrm{T}$ carriers & 33 (61.11) & $2.31(1.26-4.26)$ & 0.006 & $18(50.00)$ & $1.47(0.73-2.99)$ & 0.283 \\
\hline \multicolumn{7}{|l|}{ IL-8 -251T>A } \\
\hline $\mathrm{TT}$ & $14(25.93)$ & Reference & & $8(22.22)$ & Reference & \\
\hline TA & $29(53.70)$ & $1.49(0.74-3.01)$ & 0.267 & $22(61.11)$ & $1.98(0.83-4.68)$ & 0.117 \\
\hline AA & $11(20.37)$ & $1.53(0.63-3.69)$ & 0.342 & $6(16.67)$ & $1.46(0.47-4.51)$ & 0.509 \\
\hline A carriers & $40(74.07)$ & $1.50(0.77-2.93)$ & 0.234 & 28 (77.78) & $1.84(0.80-4.23)$ & 0.148 \\
\hline \multicolumn{7}{|c|}{ IL-10 -1082A>G } \\
\hline AA & $18(33.33)$ & Reference & & 15 (41.67) & Reference & \\
\hline AG & $27(50.00)$ & $1.18(0.61-2.29)$ & 0.625 & $16(44.44)$ & $0.84(0.39-1.80)$ & 0.651 \\
\hline GG & $9(16.67)$ & $1.31(0.53-3.22)$ & 0.560 & $5(13.89)$ & $0.87(0.29-2.60)$ & 0.804 \\
\hline G carriers & $36(66.67)$ & $1.20(0.64-2.27)$ & 0.554 & $21(58.33)$ & $0.85(0.41-1.73)$ & 0.648 \\
\hline \multicolumn{7}{|c|}{ TNF A -308 G >A } \\
\hline GG & $44(81.48)$ & Reference & & $29(80.56)$ & Reference & \\
\hline GA & $10(18.52)$ & $0.98(0.46-2.12)$ & 0.965 & $7(19.44)$ & $1.04(0.43-2.55)$ & 0.924 \\
\hline AA & $0(0.00)$ & - & & $0(0.00)$ & - & \\
\hline A carriers & $10(18.52)$ & $0.94(0.44-2.01)$ & 0.865 & $7(19.44)$ & $0.99(0.41-2.42)$ & 0.989 \\
\hline
\end{tabular}

OR, odds ratio; CI, confidence interval; TNF, tumor necrosis factor; IL, interleukin. 
A

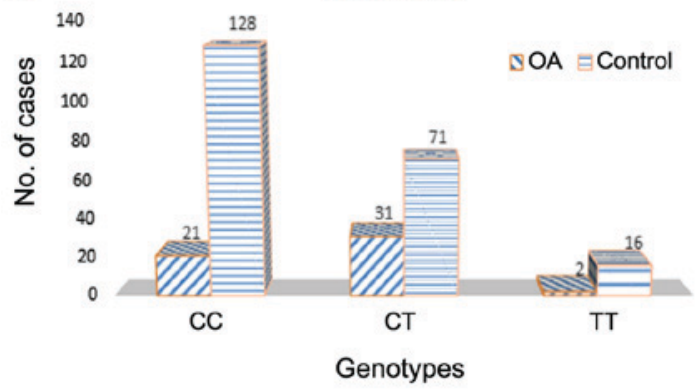

C

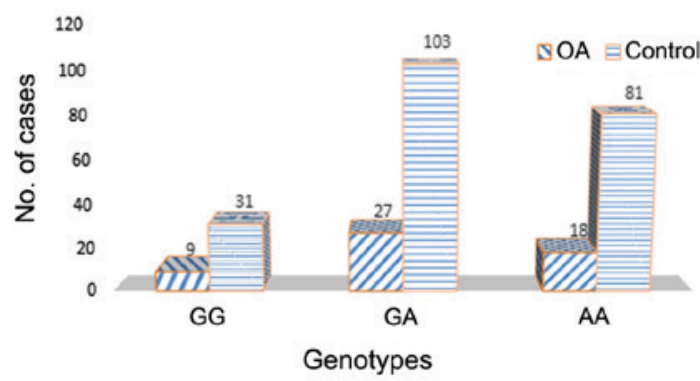

B

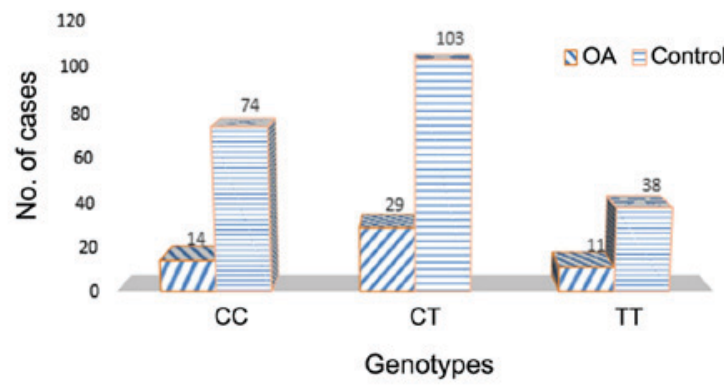

D

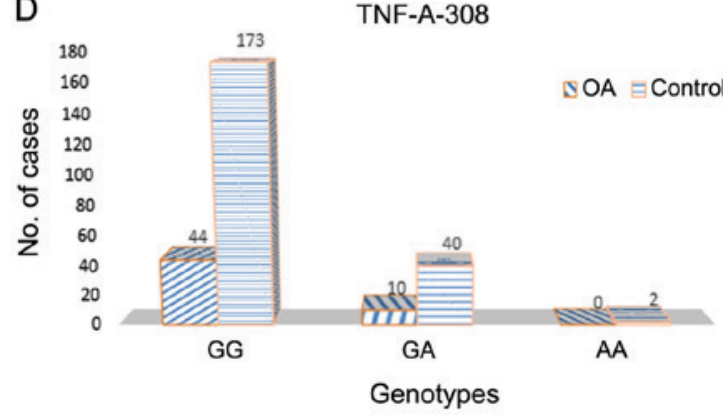

Figure 1. Genotype frequencies of IL-4R -3223C $>$ T (A), IL-8 -251T>A (B), IL-10-1082A $>$ G (C) and TNF-A -308G $>$ A (D) SNPs in controls and cases with knee OA. TNF, tumor necrosis factor; IL, interleukin; OA, osteoarthritis.
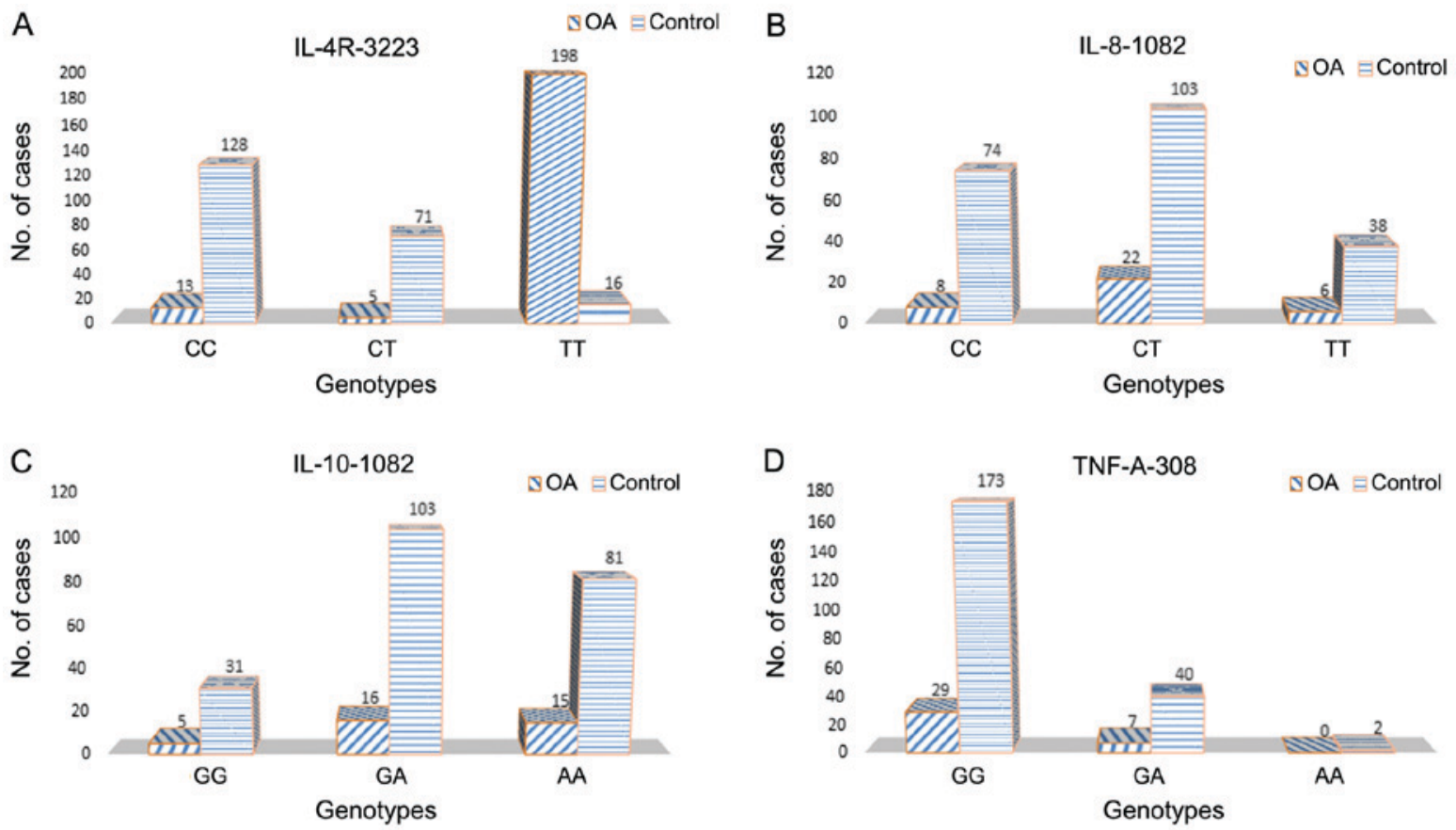

Figure 2. Genotype frequencies of IL-4R -3223C $>$ T (A), IL-8 -251T>A (B), IL-10-1082A $>$ G (C) and TNF-A -308G $>$ A (D) SNPs in controls and cases with hip OA. TNF, tumor necrosis factor; IL, interleukin; OA, osteoarthritis; SNPs, single nucleotide polymorphisms.

between IL-4R SNPs and OA susceptibility in different populations, with inconsistent results. A significant association was found for both rs1805013 and rs1805016 IL-4R SNPs and hip OA in a case-control study including UK Caucasians subjects (14). In contrast to Forster et al (14), another UK study was unable to replicate the associations of these two SNPs with hip OA and/or knee OA susceptibility (15). Furthermore, Vargiolu et al (16) suggested a positive association in an Italian population between both rs1805013 and rs1805015 located in the IL-4R gene and overall risk for hand OA, while in the subgroups analysis only the association between rs1805013 and non-erosive hand OA was significant. In addition, the possible involvement of the IL-4/IL-4R axis in the pathogenesis of OA was suggested by an association of the IL-4 intron 3 VNTR polymorphism and the incidence of knee OA in a Turkish population (17). No statistically significant associations were 
noted between IL-4R SNPs (rs1805015 and rs1805016) and hand OA in a Finnish study (18).

In the present study no correlation was noted between the IL-8 -251T>A (rs4073), IL-10 -1082A>G (rs1800896) and TNF-A -308G >A (rs1800629) SNPs and OA susceptibility. A statistically significant higher frequency of IL-8 -251TT genotype and IL-8 -251T allele was noted in patients diagnosed with OA compared with controls in a Han Chinese population, suggesting that the TT genotype and the T allele of the IL- 8 gene at position-251 confer a high risk in OA. In the same study, the IL-8 SNP located at position $+781 \mathrm{C}>\mathrm{T}$ influenced the risk of OA (19). Furthermore, IL-10 -1082A $>$ G, -819T $>C$ and $-592 \mathrm{~A}>\mathrm{C}$ gene promoter SNPs were tested in a Chinese Han population and no significant difference was observed in the allele and/or haplotype frequencies between end-stage knee OA and the controls (20). Similar results were obtained in a Dutch population, where no significant association was detected between distal interphalangeal OA and IL-10 SNPs, including IL-10 -1082A $>$ G (21). Moreover, no significant difference in the genotype distribution between OA individuals and controls was observed for the IL-10 -1082A $>$ G gene and the hand OA among Finnish women (18). A positive correlation between IL-10 G microsatellite SNP and idiopathic knee OA was noted in a Greek case-control study, and the carriers of the LL genotype were at 4 times higher risk than the SS genotype (22).

The association between TNF-A gene and OA susceptibility has been widely studied, and the published results remain inconclusive. The TNF- $\alpha$ gene encodes the important pro-inflammatory cytokine $\mathrm{TNF}-\alpha$ and different genetic variants located in the promoter region have been associated with altered gene expression, notably at the -308 position $(23,24)$. No association was observed in the present study between the TNF- $\alpha-308 \mathrm{G}>\mathrm{A}$ SNP and the risk of OA, which is consistent with the results from the Turkish Caucasian and Mexican Mestizo population. Both studies included only knee OA $(25,26)$. On the contrary, in the Han Chinese population, the TNF- $\alpha-308 \mathrm{~A}$ allele was found to increase the risk, whereas the TNF- $\alpha-238$ SNP did not play a role in OA patients (27). Similar findings were reported in the other Asian population, although the risk of OA was significantly higher for carriers of the TNF- $\alpha-308 \mathrm{~A}$ allele in a Korean Population (28). A recent meta-analysis, which included 983 OA cases and 1355 control subjects indicated a significant association between TNF- $\alpha$ $-308 \mathrm{~A}$ allele and the risk of OA. An increased OA risk was found in the recessive genetic model and higher frequency of both AA genotype and GA genotype was observed in OA patients compared with the control population. Interestingly, the association only existed among the Asian population but not among the Caucasian population (29).

The current study has some limitations. Firstly, our findings rely on a small sample and the selection bias cannot be ruled out in this hospital case-control study. OA susceptibility is influenced by multiple interactions between different other genes, which were not evaluated and additional data regarding the biological effects of investigated SNPs are required. Moreover, a subgroup analysis with regard to additional clinicopathological parameters could not be performed for this population. In conclusion, the IL-4R $-3223 \mathrm{C}>\mathrm{T}$ (rs2057768) polymorphism was correlated with
OA susceptibility, mainly for the knee OA, in the Romanian population. The present results provide new evidence for the involvement of the IL-4/IL-4R axis in the OA pathogenesis and additional well-designed large studies are required before final conclusions can be drawn.

\section{Acknowledgements}

Not applicable.

\section{Funding}

No funding was received.

\section{Availability data and materials}

The datasets used and/or analyzed during the present study are available from the corresponding author on reasonable request.

\section{Authors' contributions}

OCR,DC, MI and EB contributed to the conception of the study. OCR, AOD and ES collected the clinical data and samples. MGC, FB, SS and MI performed the experiments. MGC, FB, SS and MI performed the data analyses. OCR, DC, AOD and EB contributed significantly to manuscript preparation. OCR, DC, AOD, FB and EB wrote the manuscript. MI, FB and EB helped perform the analysis with constructive discussions. All authors read and approved the final manuscript.

\section{Ethics approval and consent to participate}

The present study was approved by the Ethics Committee of University of Medicine and Pharmacy of Craiova approved this study and a written informed consent was provided by the included participants.

\section{Patient consent for publication}

Not applicable.

\section{Competing interests}

The authors declare that they have no competing interests.

\section{References}

1. Abramson SB and Attur M: Developments in the scientific understanding of osteoarthritis. Arthritis Res Ther 11: 227, 2009.

2. Loeser RF, Goldring SR, Scanzello CR and Goldring MB: Osteoarthritis: A disease of the joint as an organ. Arthritis Rheum 64: 1697-1707, 2012.

3. Goldring MB and Goldring SR: Osteoarthritis. J Cell Physiol 213: 626-634, 2007

4. Felson DT: An update on the pathogenesis and epidemiology of osteoarthritis. Radiol Clin North Am 42: 1-9, v, 2004.

5. Zhang Y and Jordan JM: Epidemiology of osteoarthritis. Clin Geriatr Med 26: 355-369, 2010.

6. Brooks P: Inflammation as an important feature of osteoarthritis. Bull World Health Organ 81: 689-690, 2003.

7. Robinson WH, Lepus CM, Wang Q, Raghu H, Mao R, Lindstrom TM and Sokolove J: Low-grade inflammation as a key mediator of the pathogenesis of osteoarthritis. Nat Rev Rheumatol 12: 580-592, 2016. 
8. Kapoor M, Martel-Pelletier J, Lajeunesse D, Pelletier JP and Fahmi H: Role of proinflammatory cytokines in the pathophysiology of osteoarthritis. Nat Rev Rheumatol 7: 33-42, 2011.

9. Malemud CJ: Biologic basis of osteoarthritis: State of the evidence. Curr Opin Rheumatol 27: 289-294, 2015.

10. Yang F, Hu A, Zhao D, Guo L, Yang L, Wang B, Tian F, Liu B, Huang $\mathrm{S}$ and $\mathrm{Xie} \mathrm{H}$ : An insertion/deletion polymorphism at the microRNA-122 binding site in the interleukin-1alpha 3 -untranslated region is associated with a risk for osteoarthritis. Mol Med Rep 12: 6199-6206, 2015.

11. Mapp PI and Walsh DA: Mechanisms and targets of angiogenesis and nerve growth in osteoarthritis. Nat Rev Rheumatol 8 : 390-398, 2012.

12. Mabey T and Honsawek S: Cytokines as biochemical markers for knee osteoarthritis. World J Orthop 6: 95-105, 2015.

13. Burada F, Dumitrescu T, Nicoli R, Ciurea ME, Angelescu C, Mixich F and Ioana M: IL-1RN $+2018 \mathrm{~T}>\mathrm{C}$ polymorphism is correlated with colorectal cancer. Mol Biol Rep 40: 2851-2857, 2013.

14. Forster T, Chapman $\mathrm{K}$ and Loughlin J: Common variants within the interleukin 4 receptor alpha gene (IL4R) are associated with susceptibility to osteoarthritis. Hum Genet 114: 391-395, 2004.

15. Limer KL, Tosh K, Bujac SR, McConnell R, Doherty S, Nyberg F, Zhang W, Doherty M, Muir KR and Maciewicz RA: Attempt to replicate published genetic associations in a large, well-defined osteoarthritis case-control population (the GOAL study). Osteoarthritis Cartilage 17: 782-789, 2009.

16. Vargiolu M, Silvestri T, Bonora E, Dolzani P, Pulsatelli L, Addimanda O, Mancarella L, Punzi L, Fioravanti A, Facchini A, Romeo G, et al: Interleukin-4/interleukin-4 receptor gene polymorphisms in hand osteoarthritis. Osteoarthritis Cartilage 18: 810-816, 2010.

17. Yigit S, Inanir A, Tekcan A, Tural E, Ozturk GT, Kismali G and Karakus N: Significant association of interleukin-4 gene intron 3 VNTR polymorphism with susceptibility to knee osteoarthritis. Gene 537: 6-9, 2014

18. Hämäläinen $S$, Solovieva $S$, Vehmas T, Leino-Arjas $P$ and Hirvonen A: Variations in the TNFalpha gene and their interactions with the IL4R and IL10 genes in relation to hand osteoarthritis. BMC Musculoskelet Disord 15: 311, 2014.

19. He Y, Liang X, Wu X, Meng C, Wu B, Fu D, Jin S, Yang S and Wang H: Association between interleukin 8 -251 A/T and +781 $\mathrm{C} / \mathrm{T}$ polymorphisms and osteoarthritis risk. Immunol Lett 162 : 207-211,2014.
20. Lv C, Xu X, Wang J, Zhang Z, Zhang D, Guo C, Geng C and Sun Y: Combined effect of cytokine gene polymorphisms on end-stage knee osteoarthritis from Chinese Han population. Rheumatol Int 32: 3625-3629, 2012.

21. Riyazi N, Kurreeman FA, Huizinga TW, Dekker FW, Stoeken-Rijsbergen $\mathrm{G}$ and Kloppenburg M: The role of interleukin 10 promoter polymorphisms in the susceptibility of distal interphalangeal osteoarthritis. J Rheumatol 32: 1571-1575, 2005.

22. Fytili P, Giannatou E, Karachalios T, Malizos K and Tsezou A: Interleukin-10G and interleukin-10R microsatellite polymorphisms and osteoarthritis of the knee. Clin Exp Rheumatol 23: 621-627, 2005

23. Kroeger KM, Carville KS and Abraham LJ: The -308 tumor necrosis factor-alpha promoter polymorphism effects transcription. Mol Immunol 34: 391-399, 1997.

24. Uglialoro AM, Turbay D, Pesavento PA, Delgado JC, McKenzie FE, Gribben JG, Hartl D, Yunis EJ and Goldfeld AE: Identification of three new single nucleotide polymorphisms in the human tumor necrosis factor-alpha gene promoter. Tissue Antigens 52: 359-367, 1998.

25. Sezgin M, Barlas IO, Ankarali HC, Altintaş ZM, Türkmen E, Gökdoğan T, Sahin G and Erdal M: Tumour necrosis factor alpha -308G/A gene polymorphism: Lack of association with knee osteoarthritis in a Turkish population. Clin Exp Rheumatol 26: 763-768, 2008.

26. Muñoz-Valle JF, Oregón-Romero E, Rangel-Villalobos H, Martínez-Bonilla GE, Castañeda-Saucedo E, Salgado-Goytia L, Leyva-Vázquez MA, Illades-Aguiar B, Alarcón-Romero Ldel C, Espinoza-Rojo $M$ and Parra-Rojas I: High expression of TNF alpha is associated with -308 and -238 TNF alpha polymorphisms in knee osteoarthritis. Clin Exp Med 14: 61-67, 2014.

27. Ji B, Shi J, Cheng X, Zhou J, Zhou Q, Cao C and Pang J: Association analysis of two candidate polymorphisms in the tumour necrosis factor-alpha gene with osteoarthritis in a Chinese population. Int Orthop 37: 2061-2063, 2013.

28. Han L, Song JH, Yoon JH, Park YG, Lee SW, Choi YJ, Nam SW, Lee JY and Park WS: TNF- $\alpha$ and TNF- $\beta$ Polymorphisms are Associated with Susceptibility to Osteoarthritis in a Korean Population. Korean J Pathol 46: 30-37, 2012.

29. Kou S and Wu Y: Meta-analysis of tumor necrosis factor alpha -308 polymorphism and knee osteoarthritis risk. BMC Musculoskelet Disord 15: 373, 2014. 